\title{
THE HOLOMORPHY AND NONVANISHING OF NORMALIZED LOCAL INTERTWINING OPERATORS
}

\author{
YUANLI ZHANG
}

Let $\mathrm{G}$ be a connected reductive quasi-split algebraic group defined over a p-adic field $\mathbf{F}$ of characteristic zero. For $\pi$ irreducible admissible generic tempered representation of a standard Levi subgroup $M$ of $G$, we prove that the normalized intertwining operators are holomorphic and nonvanishing on a set larger than the closure of the positive chamber of $M$, under some assumptions. As an application, we prove that if $\mathrm{G}$ is a split special orthogonal group (if $\mathrm{G}$ is even orthogonal, $\mathrm{F}$ can be archimedean) and $\pi$ is an irreducible unitary representation of the Siegel Levi subgroup M of G, then the normalized intertwining operators are holomorphic and nonvanishing on a set larger than the closure of the positive chamber of M.

\section{Introduction.}

Let $\mathrm{F}$ be a local field of characteristic zero and $\mathrm{G}$ be a connected reductive quasi-split algebraic group defined over $\mathrm{F}$. We let $A_{0}$ be a maximal split torus of $\mathrm{G}$ and let $\Phi$ be the set of roots of $\mathrm{G}$ with respect to $A_{0}$. We fix a Borel subgroup of $\mathrm{G}$ which contains $A_{0}$, so we define an order on $\Phi$. We denote by $\Phi^{+}$the set of positive roots, by $\Phi^{-}$the set of negative roots, and by $\Delta$ the set of simple roots. We let $W=W\left(G, A_{0}\right)$ denote the Weyl group of $\mathrm{G}$ with respect to $A_{0}$.

Let $\theta$ be a subset of $\Delta$, we denote by $\sum_{\theta}$ the subset of $\Phi$ whose elements are $\mathbb{Z}$ - linear span of $\theta, \sum_{\theta}^{+}\left(\sum_{\theta}^{-}\right)$the intersection of $\sum_{\theta}$ and $\Phi^{+}\left(\Phi^{-}\right.$, respectively). Let $A_{\theta}$ be the connected component, where the identity lies, of $\cap_{\alpha \in \theta} \operatorname{ker} \alpha$. Then put $M_{\theta}=C_{G}\left(A_{\theta}\right)$ and $N_{\theta}=\Pi N_{\alpha}$, where $N_{\alpha}$ is the unipotent subgroup of $\mathrm{G}$ such that its Lie algebra is $\mathfrak{g}_{\alpha}$, where $\alpha$ runs over the set $\Phi^{+}-\sum_{\theta}^{+}$. Set $P_{\theta}=M_{\theta} N_{\theta}$. Then $P_{\theta}$ is the standard parabolic subgroup of G corresponding to $\theta$, and $M_{\theta}$ and $N_{\theta}$ are its Levi and unipotent radical of $P_{\theta}$, respectively. Let $\mathfrak{a}_{\theta}$ be the real Lie algebra of $A_{\theta}$, and denote by $\mathfrak{a}_{\theta, \mathbb{C}}$ its complexification. Let $\mathfrak{a}_{\theta}^{*}$ and $\mathfrak{a}_{\theta, \mathbb{C}}^{*}$ be the duals of $\mathfrak{a}_{\theta}$ and $\mathfrak{a}_{\theta, \mathbb{C}}$, respectively. More precisely,

$$
\mathfrak{a}_{\theta}=\operatorname{Hom}_{\mathbb{Z}}\left(X\left(M_{\theta}\right)_{F}, \mathbb{R}\right), \mathfrak{a}_{\theta}^{*}=X\left(M_{\theta}\right)_{F} \otimes_{\mathbb{Z}} \mathbb{R},
$$


where the $X\left(M_{\theta}\right)_{F}$ is the $\mathbb{Z}-$ module of the F-rational characters of $M_{\theta}$. Since the one parameter subgroups of $A_{\theta}$ can be identified with a subset of $\mathfrak{a}_{\theta}$ which generates $\mathfrak{a}_{\theta}$, we fix the duality $\langle\cdot, \cdot\rangle$ on the $\mathfrak{a}_{\theta, \mathbb{C}}^{*}$ and $\mathfrak{a}_{\theta, \mathbb{C}}$ corresponding the duality between $X\left(A_{\theta}\right)_{F}$ and the one parameter subgroups of $A_{\theta}$.

Now let $H_{\theta}$ be the Harish-Chandra map from $M_{\theta}$ to $\mathfrak{a}_{\theta}$ defined by

$$
q^{\left\langle H_{\theta}(m), \chi\right\rangle}=|\chi(m)|_{F}, \quad \text { for } m \in M_{\theta}, \quad \chi \in X\left(M_{\theta}\right)_{F},
$$

where $q$ is the cardinality of the residue field of $\mathrm{F}$, when $\mathrm{F}$ is nonarchimedean, and is exp, when $\mathrm{F}$ is archimedean and $|\cdot|_{F}$ is the absolute value of $\mathrm{F}$.

We shall use the same notation $G$ for the group of the F-rational points of $\mathrm{G}$, if there is no confusion.

Given an irreducible admissible representation $\pi$ of $M_{\theta}$, and $\nu \in \mathfrak{a}_{\theta, \mathbb{C}}^{*}$, we use the notation $I(\nu, \pi, \theta)$ to denote the parabolically induced representation of $G$, i.e.,

$$
I(\nu, \pi, \theta)=\operatorname{Ind}_{M_{\theta} N_{\theta}}^{G} \pi \otimes q^{\left\langle\nu, H_{\theta}(\cdot)\right\rangle} \otimes 1_{N_{\theta}},
$$

which is normalized so that every irreducible unitary representation of $M_{\theta}$ is induced to a unitary one of G for imaginary $\nu$.

For the given data in the above, one defines the standard intertwining operator $A(w, \pi, \theta, \nu)$ which intertwines the induced representations $I(\nu, \pi, \theta)$ and $I(w \nu, w \pi, w \theta)$ when $\nu$ is not a pole of $A(w, \pi, \theta, \nu)$. For global reasons, it is important to normalize these operators by root numbers and L-functions as conjectured by Langlands [4]. When $\mathrm{F}$ is archimedean or $\pi$ is generic, this is done in [1] and [7], respectively. For definitions and some descriptions of standard intertwining operators and normalized intertwining operators, respectively, see Section 1.

If $\pi$ is tempered and $\operatorname{Re} \nu$ is in the open positive chamber of $\mathfrak{a}_{\theta}^{*}$, then $N(w, \pi, \theta, \nu)$ is expected to be holomorphic and nonvanishing (cf. Conjecture A below). Suppose $\pi$ is tempered. In this paper we prove the holomorphy and nonvanishing of $N(w, \pi, \theta, \nu)$ for $\nu$ with $\operatorname{Re} \nu$ in a subset of $\mathfrak{a}_{\theta}^{*}$ which is larger than the closure of the open positive chamber of $\mathfrak{a}_{\theta}^{*}$, under both Conjecture A (Conjecture 7.1 of [7]) and Assumption A, when F is p-adic. This is our Theorem 3. One expects this assumption to be true, as it is evident from the recent work of Casselman and Shahidi [3]. (See the remark before Theorem 2.) For $\mathrm{F}$ archimedean and $\mathrm{G}=\mathrm{SO}(2 \mathrm{n})$, what we need is proved in [3]. For the result of [3], see Theorem 2 in Section 2 of this paper. We then apply Theorem 3 to the split special orthogonal groups $\mathrm{G}=\mathrm{SO}(2 \mathrm{n})$ and $\mathrm{SO}(2 \mathrm{n}+1)$, their standard Siegel Levi subgroup $M_{\theta}$, and $\pi$ an irreducible unitary generic representation of $M_{\theta}$. This is our Theorem 4 . The classification result on the unitary dual of GL(n) due to Vogan [12] and Tadic [10] is used in the proof of Theorem 4. Our result is quite similar 
to that for GL(n) in [5]. We hope that this result will also have a similar application in the study of the holomorphy of the exterior square L-function of an irreducible automorphic cuspidal representation of GL(n) as in [5].

I would like to thank Professor F. Shahidi for suggesting the problem of this paper to me, for many conversations from which I have learned about representation theory and Automorphic L-functions, and for his careful reading and correcting the manuscript of this paper. Also I would like to thank Professor D. Goldberg for helpful conversations.

\section{Normalized Intertwining Operators.}

Given an irreducible admissible representation $\pi$ of $M_{\theta}, \nu \in a_{\theta, \mathbb{C}}^{*}$, and $w \in W$, such that $w(\theta) \subset \Delta$. Fix a representative of $w$ in $\mathrm{G}$ which we also denote by $w$. One can define the standard intertwining operator by

$$
A(\nu, \pi, \theta, w) f(g)=\int_{U \cap w N_{\theta}^{-} w^{-1}} f\left(w^{-1} n g\right) d n,
$$

here $f$ is in the space of $I(\nu, \pi, \theta), U$ is the unipotent radical of the fixed Borel subgroup B, $N_{\theta}^{-}$is the unipotent radical subgroup opposite to $N_{\theta}$, the measure in the integral is a fixed Haar measure of $N_{\theta}$. It is known that the integral absolutely converges when the real part of $\nu$ is big enough, and this operator has an analytic continuation to a meromorphic function on $\mathfrak{a}_{\theta, \mathbb{C}}^{*}$. When $\nu$ is not a pole, $A(\nu, \pi, \theta, w)$ is really an intertwining operator from $I(\nu, \pi, \theta)$ to $I(w \nu, w \pi, w \theta)$ in the usual sense. These operators play a very important role in the study of representations as well as automorphic L-functions. For example, the location of poles of this operator determines both the reducibility of the induced representation $I(\nu, \pi, \theta)$ and the poles of certain local L-functions, c.f. [3]. We can define global standard intertwining operators similarly. These global intertwining operators are necessary for the decomposition of the spectrum of automorphic forms and the analytic properties of automorphic L-functions. The $G L(n)$ situation is well known, see [5] for example.

Langlands [4] suggested that one could define the normalizing factors essentially by the quotients of the L-functions. For the archimedean case, the L-functions are defined by Artin L-functions, see [11]. In this case, Arthur [1] proved that the normalizing factors defined following Langlands' suggestion satisfy all the expected properties. When F is nonarchimedean, Shahidi [7] defined the L-functions for irreducible admissible generic representations of $M_{\theta}$ and proved the decomposition property of the normalized intertwining operators, see Theorem 1 later.

Let us describe the normalizing factor $r(\nu, \pi, \theta, w)$ for $\pi$ generic. We first assume that $\theta$ is maximal in $\Delta$, say $\theta=\Delta-\{\alpha\}$, and $w=w_{l} w_{l, \theta}$. In this 
paper for $\theta \subset \Delta$, we denote $w_{l, \theta}$ the longest element of the subgroup $W_{\theta}$ of $\mathrm{W}$ which is generated by the reflections corresponding to the elements in $\theta$, and $w_{l}=w_{l, \Delta}$. Let $r_{\theta}$ be the adjoint representation of the L-group of $M_{\theta}$ on the Lie algebra of the L-group of $N_{\theta}$. Then

$$
r_{\theta}=r_{\theta}^{(1)} \oplus \cdots \oplus r_{\theta}^{(m)},
$$

where each $r_{\theta}^{(i)}$ is an irreducible representation of the L-group of $M_{\theta}$ and indexed as in [8]. We call $l\left(r_{\theta}\right)=m$ the length of $r_{\theta}$. Then define

$$
r(\nu, \pi, \theta, w)=\prod_{i=1}^{m} \frac{L\left(i\left\langle\nu, \alpha^{\vee}\right\rangle, \tilde{\pi}, r_{\theta}^{(i)}\right)}{L\left(1+i\left\langle\nu, \alpha^{\vee}\right\rangle, \tilde{\pi}, r_{\theta}^{(i)}\right) \epsilon\left(i\left\langle\nu, \alpha^{\vee}\right\rangle, \pi, r_{\theta}^{(i)}, \psi_{F}\right)},
$$

where the L-functions and $\epsilon$ - factors were defined by Shahidi in [7], $\alpha^{\vee}$ is the standard coroot corresponding to root $\alpha$, and the $\psi_{F}$ is a fixed non-trivial additive character of $\mathrm{F}$.

Before we define the normalizing factors for non-maximal $\theta$, let us recall the procedure by which one can decompose a standard intertwining operator into a product of rank one operators.

Proposition 1 (Langlands [6, p. 14], Shahidi [9]). Let $\theta \subset \Delta, w \in W$ such that $w \theta \subset \Delta$. Then there is a finite sequence $\alpha_{1}, \alpha_{2}, \cdots, \alpha_{n}$ in $\Delta$, such that if we put $\Omega_{i}=\theta_{i} \cup\left\{\alpha_{i}\right\}, \theta_{i+1}=w_{i} \theta_{i}, w_{i}=w_{l, \Omega_{i}} w_{l, \theta_{i}}, \bar{w}_{i}=w_{i-1} \cdots w_{1}$, for $1<i \leq n-1$ and $\theta_{1}=\theta$, then

(1) $w=w_{n} w_{n-1} \cdots w_{1}, \theta_{n}=w \theta$, and $n$ is the length of $w$,

(2) $A(\nu, \pi, \theta, w)=A\left(\nu_{n-1}, \pi_{n-1}, \theta_{n-1}, w_{n-1}\right) \cdots A\left(\nu_{1}, \pi_{1}, \theta_{1}, w_{1}\right)$ where $\nu_{i}=w_{i-1}\left(\nu_{i-1}\right), \pi_{i}=w_{i-1}\left(\pi_{i-1}\right)$, for $i=2, \cdots, n-1$, and $\nu_{1}=\nu, \pi_{1}=\pi$.

Remark. Let $\Phi\left(A_{\theta}, G\right)$ be the set of roots of $\mathrm{G}$ with respect to $A_{\theta}$. Then $\Phi\left(A_{\theta}, G\right)$ can be identified with the set $\Phi-\sum_{\theta} \bmod$ the restriction of the elements of $\Phi$ to $A_{\theta}$. Let $\Phi^{+}\left(A_{\theta}, G\right)\left(\Phi^{-}\left(A_{\theta}, G\right)\right.$, respectively) be the subset of $\Phi\left(A_{\theta}, G\right)$ which are restrictions of the elements in $\Phi^{+}\left(\Phi^{-}\right.$, respectively). Let $\Phi_{r}\left(A_{\theta}, G\right)$ be the set of reduced roots of $\Phi\left(A_{\theta}, G\right)$. If we let

$$
\Phi_{r}^{+}(\theta, w)=\left\{\alpha \in \Phi_{r}^{+}\left(A_{\theta}, G\right) ; w \alpha \in \Phi^{-}\right\},
$$

then

$$
\Phi_{r}^{+}(\theta, w)=\left\{\beta_{1}, \beta_{2}, \ldots, \beta_{n}\right\}
$$

where $\beta_{i}=\bar{w}_{i}^{-1} \alpha_{i}$ for $1 \leq i \leq n$. For this see which we already used in the statement of Proposition 1. 
Now we put

$$
\begin{aligned}
r(\nu, \pi, \theta, w) & =\prod_{i=1}^{n-1} r\left(\nu_{i}, \pi_{i}, \theta_{i}, w_{i}\right), \\
N(\nu, \pi, \theta, w) & =r(\nu, \pi, \theta, w)^{-1} A(\nu, \pi, \theta, w) .
\end{aligned}
$$

We call $N(\nu, \pi, \theta, w)$ the normalized intertwining operator.

Remark. By the remark following Proposition 1, we can see that the definition of $r(w, \pi, \theta, \nu)$ does not depend on the choice of the decomposition of $w$ described in Proposition 1. Indeed, suppose we have two decompositions according to

$$
\begin{aligned}
& \alpha_{1}, \alpha_{2}, \ldots, \alpha_{n}, w=w_{n} \cdots w_{1} \\
& \alpha_{1}^{\prime}, \alpha_{2}^{\prime}, \ldots, \alpha_{n}^{\prime}, w=w_{n}^{\prime} \cdots w_{1}^{\prime}
\end{aligned}
$$

respectively, as in Proposition 1. By the remark, we can assume $\bar{w}_{i}^{-1} \alpha_{i}=$ ${\overline{w^{\prime}}}_{k}^{\prime-1} \alpha_{k}^{\prime}=\beta$ for some $\beta \in \Phi_{r}^{+}(\theta, w)$. Then if we let $\bar{w}=\bar{w}_{i} \bar{w}_{k}^{\prime-1}$, then $\alpha_{i}=\bar{w} \alpha_{k}^{\prime}, \nu_{i}=\bar{w} \nu_{k}^{\prime}, \pi_{i}=\bar{w} \pi_{k}^{\prime}, \theta_{i}=\bar{w} \theta_{k}^{\prime}$. So we must have

$$
r\left(\nu_{i}, \pi_{i}, \theta_{i}, w_{i}\right)=r\left(\nu_{k}^{\prime}, \pi_{k}^{\prime}, \theta_{k}^{\prime}, w_{k}^{\prime}\right) .
$$

From this we see the claim.

The normalized intertwining operators also have analytic continuation to meromorphic functions on $\mathfrak{a}_{\theta, \mathbb{C}}^{*}$, as the standard intertwining operators do. But they satisfy more properties than standard intertwining operators. One of the most important properties which is not satisfied by standard intertwining operators is the analogue of (2) of Proposition 1 for an arbitrary decomposition of $w$. We record this in the following theorem.

Theorem 1 (Arthur [1], Shahidi [7]). Let $\pi$ be an irreducible admissible generic representation of $M_{\theta}, w_{1} \theta, w_{2} w_{1} \theta \subset \Delta, \nu \in a_{\theta, \mathbb{C}}^{*}$. Then by suitably choosing a Haar measure for the standard intertwining operators, we have

$$
N\left(\nu, \pi, \theta, w_{2} w_{1}\right)=N\left(w_{1} \nu, w_{1} \pi, w_{1} \theta, w_{2}\right) N\left(\nu, \pi, \theta, w_{1}\right) .
$$

\section{The Holomorphy and Nonvanishing of the Normalized Intertwining Operators.}

In this section, we prove our main theorem, i.e., Theorem 3.

We first state a conjecture due to Shahidi [7] and an assumption for our theorem. 
Conjecture A (Shahidi [7]). Let $F$ be a nonarchimedean field of characteristic zero and $\theta=\Delta-\{\alpha\}$. If $\pi$ is an irreducible tempered generic representation of $M_{\theta}$, then $L\left(\left\langle\nu, \alpha^{\vee}\right\rangle, \pi, r_{\theta}\right)$ is holomorphic for $\nu \in \mathfrak{a}_{\theta, \mathbb{C}}^{*}$ satisfying $\left\langle\operatorname{Re} \nu, \alpha^{\vee}\right\rangle>0$.

Remark. This conjecture was proved in many cases by Shahidi in [7]. For example, if $l\left(r_{\theta}\right)=m=1$ or $\pi$ is supercuspidal, the conjecture is true. The case of classical groups is proved in [3]. If $\mathrm{F}$ is archimedean, the conclusion of the conjecture is true, even if we remove the generic condition on $\pi$, see [1].

Assumption A. Let $\mathrm{F}$ be a p-adic field, $\theta=\Delta-\{\alpha\}$, and $m=l\left(r_{\theta}\right)$. If $\pi$ is an irreducible tempered generic representation of $M_{\theta}$, then $I(\nu, \pi, \theta)$ is irreducible for every $\nu \in \mathfrak{a}_{\theta, \mathbb{C}}^{*}$ satisfying $-\frac{1}{m}<\left\langle\operatorname{Re} \nu, \alpha^{\vee}\right\rangle<0$.

Remark. If $\pi$ is a supercuspidal representation of $M_{\theta}$, then the conclusion in Assumption A is true. This was proved by Shahidi in [9]. Moreover, it is proved in [3] that if standard modules of generic representations are irreducible (Vogan's theorem in the real case), then the points of reducibility in the negative Weyl chamber will have their real parts not larger than $-1 / m$, confirming our Assumption A.

Now we record a recent result due to Casselman and Shahidi.

Theorem 2 (Casselman and Shahidi, [3]). If $F$ is archimedean, $G=$ $S O(2 n)$ and $M_{\theta}$ is the Siegel-Levi subgroup of $G$, then

$$
\frac{1}{L\left(\left\langle\nu, \alpha^{\vee}\right\rangle, \tilde{\pi}, r_{\theta}\right)} A\left(\nu, \pi, \theta, w_{0}\right)
$$

is entire on $\nu \in \mathfrak{a}_{\theta, \mathbb{C}}^{*}$ for every irreducible admissible generic representation $\pi$ of $M_{\theta}$.

Lemma 1. Let $\theta$ and $\pi$ be as in Assumption A, and $w=w_{l} w_{l, \theta}$. Assume Conjecture A and Assumption A. Then $N(\nu, \pi, \theta, w)$ is holomorphic for $\nu \in \mathfrak{a}_{\theta, \mathbb{C}}^{*}$ satisfying $-\frac{1}{m}<\left\langle\operatorname{Re} \nu, \alpha^{\vee}\right\rangle<0$.

Proof. Since Re $\nu$ is in the negative chamber of $\mathfrak{a}_{\theta}^{*}$, Re $w \nu$ is in the positive chamber of $\mathfrak{a}_{w \theta}^{*}$. So

$$
N\left(w \nu, w \pi, w \theta, w^{-1}\right)
$$

is holomorphic, since both the L-functions and the standard intertwining operator are holomorphic under Conjecture A. Conjecture A also implies that $N\left(w \nu, w \pi, w \theta, w^{-1}\right)$ and $A\left(w \nu, w \pi, w \theta, w^{-1}\right)$ have the same image and the same kernel. So $N\left(w \nu, w \pi, w \theta, w^{-1}\right)$ is an isomorphism of the vector 
spaces of $I(\nu, \pi, \theta)$ and $I(w \nu, w \pi, w \theta)$ if and only if $I(\nu, \pi, \theta)$ is irreducible. When $N\left(w \nu, w \pi, w \theta, w_{-1}\right)$ is isomorphic, its inverse is $N(\nu, \pi, \theta, w)$, hence $N(\nu, \pi, \theta, w)$ must be holomorphic.

Lemma 2. Let $\theta$ and $\pi$ be as in Assumption $\mathrm{A}$, and $w=w_{l} w_{l, \theta}$. Then $N(\nu, \pi, \theta, w)$ is holomorphic for all $\nu \in \mathfrak{a}_{\theta, \mathbb{C}}^{*}$ satisfying $\left\langle\operatorname{Re} v, \alpha^{\vee}\right\rangle=0$.

Proof. Recall that

$$
\begin{aligned}
& N(\nu, \pi, \theta, w) \\
& =\prod_{i=1}^{m} \frac{L\left(1+i\left\langle\operatorname{Re} \nu, \alpha^{\vee}\right\rangle, \tilde{\pi}, r_{\theta}^{(i)}\right)}{L\left(i\left\langle\operatorname{Re} \nu, \alpha^{\vee}\right\rangle, \tilde{\pi}, r_{\theta}^{(i)}\right)} \epsilon\left(i\left\langle\operatorname{Re} \nu, \alpha^{\vee}\right\rangle, \tilde{\pi}, r_{\theta}^{(i)}\right) A(\nu, \pi, \theta, w) .
\end{aligned}
$$

Then $\left\langle\operatorname{Re} \nu, \alpha^{\vee}\right\rangle=0$ implies that

$$
L\left(1+i\left\langle\operatorname{Re} \nu, \alpha^{\vee}\right\rangle, \tilde{\pi}, r_{\theta}^{(i)}\right)
$$

is holomorphic for $1 \leq i \leq m$. Therefore what we need to prove is that

$$
\prod_{i=1}^{m} \frac{1}{L\left(i\left\langle\operatorname{Re} \nu, \alpha^{\vee}\right\rangle, \tilde{\pi}, r_{\theta}^{(i)}\right)} A(\nu, \pi, \theta, w)
$$

is holomorphic.

Suppose that $\pi$ is in the discrete series. If $\nu$ with $\left\langle\operatorname{Re} \nu, \alpha^{\vee}\right\rangle=0$ is a pole of $A(\nu, \pi, \theta, w)$, then $I(\nu, \pi, \theta)$ is irreducible. Thus the local coefficient $C_{\psi}(\nu, \pi, \theta, w)$ defined in [9] has a zero at $\nu$ of the same order. Recall that (Theorem 3.5 of [7]), up to a non-zero constant,

$$
C_{\psi}(\nu, \pi, \theta, w)=\prod_{i=1}^{m} \frac{L\left(1-i\left\langle\operatorname{Re} \nu, \alpha^{\vee}\right\rangle, \pi, r_{\theta}^{(i)}\right)}{L\left(i\left\langle\operatorname{Re} \nu, \alpha^{\vee}\right\rangle, \tilde{\pi}, r_{\theta}^{(i)}\right)} \epsilon\left(i\left\langle\operatorname{Re} \nu, \alpha^{\vee}\right\rangle, \pi, r_{\theta}^{(i)}\right) .
$$

Every $L\left(1-i\left\langle\operatorname{Re} \nu, \alpha^{\vee}\right\rangle, \tilde{\pi}, r_{\theta}^{(i)}\right)$ is holomorphic at $\nu$, since $\left\langle\operatorname{Re} \nu, \alpha^{\vee}\right\rangle=0$. Consequently

$$
\prod_{i=1}^{m} \frac{1}{L\left(i\left\langle\operatorname{Re} \nu, \alpha^{\vee}\right\rangle, \tilde{\pi}, r_{\theta}^{(i)}\right)}
$$

has a zero of the same order. Therefore

$$
\prod_{i=1}^{m} \frac{1}{L\left(i\left\langle\operatorname{Re} \nu, \alpha^{\vee}\right\rangle, \tilde{\pi}, r_{\theta}^{(i)}\right)} A(\nu, \pi, \theta, w)
$$

is holomorphic at $\nu$. 
If $\nu$ is not a pole of $A(\nu, \pi, \theta, w)$, then $\nu$ is not a zero of $C_{\psi}(\nu, \pi, \theta, w)$, hence $N(\nu, \pi, \theta, w)$ is holomorphic at $\nu$.

Now suppose that $\pi$ is tempered. Then $\pi$ is induced from an irreducible representation in discrete series of a smaller Levi subgroup. Since the normalized intertwining operators are compatible with inducing in stages, the lemma follows.

Let $n$ be the length of $w$ and let

$$
m=\max \left\{l\left(r_{\theta_{i}}\right) ; 1 \leq i \leq n-1\right\}
$$

\section{Theorem 3.}

(1) Assume that $F$ is a p-adic field. Let $\theta \subset \Delta, \pi$ be an irreducible generic tempered representation of $M_{\theta}$, and $\nu \in \mathfrak{a}_{\theta, \mathbb{C}}^{*}, w \in W$ such that $w \theta \subset$ $\Delta$. Assume Conjecture $A$ and Assumption A are true for $G$. Then $N(\nu, \pi, \theta, w)$ is holomorphic and nonvanishing at $\nu$, if $\left.\left\langle\operatorname{Re} \nu, \alpha^{\vee}\right\rangle\right\rangle$ $-\frac{1}{m}$ for every $\alpha \in \Phi_{r}^{+}(\theta, w)$.

(2) Assume that $F$ is an archimedean field. Let $G=S O(2 n)$ and assume $M_{\theta}$ is the Siegel-Levi subgroup of $G$. Then the conclusion of (1) is true.

Proof. Without affecting the result we can assume that $\nu \in \mathfrak{a}_{\theta}^{*}$. For holomorphy of $N(\nu, \pi, \theta, w)$, we write

$$
N(\nu, \pi, \theta, w)=\prod_{i=1}^{n-1} N\left(\nu_{i}, \pi_{i}, \theta_{i}, w_{i}\right)
$$

as a composition of factors of rank one as before. We need to prove that every factor is holomorphic. By Lemma 1, Lemma 2, and the result of Casselman and Shahidi [3], what we should check is that for $1 \leq i \leq n-1$ the L-function

$$
L\left(1+j\left\langle\nu_{i}, \alpha_{i}^{\vee}\right\rangle, \pi_{i}, r_{\theta_{i}}\right)
$$

is holomorphic at $\nu_{i}$, for $1 \leq j \leq l\left(r_{\theta_{i}}\right)$, since the $\epsilon$-factors are monomials in the $q^{\left\langle\nu_{i}, \alpha_{i}^{\vee}\right\rangle}$ for $1 \leq i \leq n-1$. Now, since $\left\langle\nu_{i}, \alpha_{i}^{\vee}\right\rangle=\left\langle\nu, \beta_{i}^{\vee}\right\rangle$ for some $\beta_{i} \in \Phi_{r}^{+}(\theta, w),\left\langle\nu_{i}, \alpha_{i}^{\vee}\right\rangle>-\frac{1}{m}$. So Conjecture A implies that $N\left(\nu_{i}, \pi_{i}, \theta_{i}, w_{i}\right)$ is holomorphic at $\nu_{i}$.

For the nonvanishing of $N(\nu, \pi, \theta, w)$, we first assume that $\left\langle\nu, \alpha^{\vee}\right\rangle>0$ for every $\alpha \in \Phi_{r}^{+}(\theta, w)$. In this situation, the proposition is true under Conjecture $\mathrm{A}$, since both the normalizing factor and the standard intertwining operator are holomorphic and nonvanishing at $\nu$. 
Then we assume that $\nu$ is in the closure of the open positive chamber of $\mathfrak{a}_{\theta}^{*}$, i.e. $\nu$ satisfies that $\left\langle\nu, \alpha^{\vee}\right\rangle \geq 0$ for every $\alpha \in \Phi^{+}-\sum_{\theta}^{+}$. Let

$$
\begin{aligned}
R_{0} & =\left\{\alpha \in \Phi^{+}-\sum_{\theta}^{+} ;\left\langle\nu, \alpha^{\vee}\right\rangle=0\right\}, \\
\theta^{\prime} & =\theta \cup\left(\Delta \cap R_{0}\right) .
\end{aligned}
$$

Then $\theta \subset \theta^{\prime}$, and for any $\alpha \in \Phi^{+}-\sum_{\theta^{\prime}}^{+}$, we have $\left\langle\nu, \alpha^{\vee}\right\rangle>0$.

If $\theta^{\prime}=\Delta$, since for $1 \leq i \leq n$

$$
N\left(\nu_{i}, \pi_{i}, \theta_{i}, 1\right)=N\left(w_{i} \nu_{i}, w_{i} \pi_{i}, w_{i} \theta, w_{i}^{-1}\right) N\left(\nu_{i}, \pi_{i}, \theta_{i}, w_{i}\right)
$$

and every normalized intertwining operator in the equality above is holomormorphic and the left one is the identity operator, so $N\left(\nu_{i}, \pi_{i}, \theta_{i}, w_{i}\right)$ must have zero kernel. Therefore

$$
N(\nu, \pi, \theta, w)
$$

must be nonvanishing.

If $\theta^{\prime} \neq \Delta$, we let $w_{0}^{\prime}=w_{l} w_{l, \theta^{\prime}}, \tilde{w}=w_{0}^{\prime} w^{-1}$. Then $w_{0}^{\prime}=\tilde{w} w$, hence

$$
N\left(\nu, \pi, \theta, w_{0}^{\prime}\right)=N(w \nu, w \pi, w \theta, \tilde{w}) N(\nu, \pi, \theta, w) .
$$

Since $\nu$ is in the closure of the positive chamber of $\mathfrak{a}_{\theta}^{*}, N\left(\nu, \pi, \theta, w_{0}^{\prime}\right)$ and $N(\nu, \pi, \theta, w)$ are holomorphic at $\nu$. We claim that $N(w \nu, w \pi, w \theta, \tilde{w})$ is also holomorphic at $w \nu$. Observe that $\left\langle w \nu, \alpha^{\vee}\right\rangle=\left\langle\nu,\left(w^{-1} \alpha\right)^{\vee}\right\rangle$. For any $\alpha \in$ $\Phi^{+}(w \theta, \tilde{w})$, it is obvious that $w^{-1} \alpha \notin \sum_{\theta}$ and $\tilde{w} \alpha=w_{0}^{\prime}\left(w^{-1} \alpha\right)$. Note that $w_{0}^{\prime}=w_{l} w_{l, \theta^{\prime}}$ sends every element of $\Phi^{+}-\sum_{\theta^{\prime}}^{+}$to $\Phi^{-}$and one of $\Phi^{-}-\sum_{\theta^{\prime}}^{-}$ to $\Phi^{+}$. If $w^{-1} \alpha \in \Phi^{+}$, then $\left\langle\nu, w^{-1} \alpha\right\rangle \geq 0$. Suppose that $w^{-1} \alpha \in \Phi^{-}$. If $w^{-1} \alpha \in \Phi^{-}-\sum_{\theta^{\prime}}^{-}$, then $\tilde{w} \alpha \in \Phi^{+}$, which contradicts $\tilde{w} \alpha \in \Phi^{-}$. If $w^{-1} \alpha \in$ $\sum_{\theta^{\prime}}^{-}$, then $\left\langle\nu, w^{-1} \alpha\right\rangle=0$. Therefore, $\left\langle\nu, w^{-1} \alpha\right\rangle \geq 0$ holds for every $\alpha \in$ $\Phi^{+}(w \theta, \tilde{w})$. Hence $N(w \nu, w \pi, w \theta, \tilde{w})$ is holomorphic at $w \nu$. We also claim that $N\left(\nu, \pi, \theta, w_{0}^{\prime}\right)$ is nonvanishing at $\nu$. Indeed, for every $\alpha \in \Phi^{+}\left(\theta, w_{0}^{\prime}\right)$, if $\alpha \in \sum_{\theta^{\prime}}^{+}$then $w_{0}^{\prime} \alpha \in \Phi^{+}$which is a contradiction, since $w_{0}^{\prime} \alpha \in \Phi^{-}$. So we must have that $\alpha \in \Phi^{+}-\sum_{\theta^{\prime}}^{+}$, hence $\left\langle\nu, \alpha^{\vee}\right\rangle>0$ by the choice of $\theta^{\prime}$. Therefore $N\left(\nu, \pi, \theta, w_{0}^{\prime}\right)$ is nonvanishing at $\nu$. This together with the holomorphy of $N(w \nu, w \pi, w \theta, \tilde{w})$ and $N(\nu, \pi, \theta, w)$ implies the nonvanishing of $N(\nu, \pi, \theta, w)$ at $\nu$.

Now we prove that $N(\nu, \pi, \theta, w)$ is nonvanishing at $\nu$, if $\left\langle\nu, \alpha^{\vee}\right\rangle>-\frac{1}{m}$ for every $\alpha \in \Phi_{r}^{+}(\theta, w)$. We can find a $\tau \in W\left(G, A_{0}\right)$ such that $\tau \theta \subset \Delta$, and $\tau \nu$ is in the closure of the open positive chamber of $\mathfrak{a}_{\tau \theta}^{*}$. For the proof of this, see p. 16 of [6]. Then

$$
N\left(\tau \nu, \tau \pi, \tau \theta, w \tau^{-1}\right)=N(\nu, \pi, \theta, w) N\left(\tau \nu, \tau \pi, \tau \theta, \tau^{-1}\right)
$$


$N\left(\tau \nu, \tau \pi, \tau \theta, w \tau^{-1}\right)$ is holomorphic and nonvanishing at $\tau \nu$, by the last paragraph. Both $N\left(\tau \nu, \tau \pi, \tau \theta, \tau^{-1}\right)$ and $N(\nu, \pi, \theta, w)$ are holomorphic at $\nu$, by the above. Therefore $N(\nu, \pi, \theta, w)$ is nonvanishing at $\nu$.

Remark. From the last paragraph of the proof of the proposition, we can see that for $\pi$ irreducible tempered and generic, if $N(\nu, \pi, \theta, w)$ is holomorphic at $\nu$, then $N(\nu, \pi, \theta, w)$ is nonvanishing at $\nu$ only under Conjecture A and without appealing to Assumption A.

\section{Examples: $\mathrm{SO}(2 \mathrm{n})$ and $\mathrm{SO}(2 \mathrm{n}+1)$.}

In this section we apply the theorem of the last section to the case of split special orthogonal groups $\mathrm{SO}(2 \mathrm{n})$ and $\mathrm{SO}(2 \mathrm{n}+1)$ defined over $F$.

Let $\mathrm{G}=\mathrm{SO}(2 \mathrm{n})$ or $\mathrm{SO}(2 \mathrm{n}+1)$. We fix a maximal split torus $A_{0}$ of $\mathrm{G}$. If $\mathrm{G}=\mathrm{SO}(2 \mathrm{n})$,

$$
A_{0}=\left\{\operatorname{diag}\left(x_{1}, x_{2}, \ldots, x_{n}, x_{n}^{-1}, x_{n-1}^{-1}, \ldots, x_{1}^{-1}\right), ; x_{i} \in F^{\times}, 1 \leq i \leq n\right\},
$$

while for $\mathrm{G}=\mathrm{SO}(2 \mathrm{n}+1)$,

$$
A_{0}=\left\{\operatorname{diag}\left(x_{1}, x_{2}, \cdots, x_{n}, 1, x_{n}^{-1}, x_{n-1}^{-1}, \cdots, x_{1}^{-1}\right), ; x_{i} \in F^{\times}, 1 \leq i \leq n\right\} .
$$

Let $e_{i} \in X\left(A_{0}\right)_{F}$ be defined by, for $x \in A_{0}$

$$
e_{i}(x)=x_{i}, \text { for } 1 \leq i \leq n .
$$

Then the set of positive roots of $\mathrm{G}$ with respect to $A_{0}$ is

$$
\Phi^{+}= \begin{cases}\left\{e_{i} \pm e_{j}, ; 1 \leq i<j \leq n\right\}, & \text { if } \mathrm{G}=\mathrm{SO}(2 \mathrm{n}) \\ \left\{e_{i} ; 1 \leq i \leq n\right\} \cup\left\{e_{i} \pm e_{j} ; 1 \leq i<j \leq n\right\} & \text { if } \mathrm{G}=\mathrm{SO}(2 \mathrm{n}+1) .\end{cases}
$$

If we let

$$
\begin{aligned}
\alpha_{i} & =e_{i}-e_{i+1}, \quad 1 \leq i \leq n-1 \\
\alpha_{n} & = \begin{cases}e_{n-1}+e_{n} & \text { if } \mathrm{G}=\mathrm{SO}(2 \mathrm{n}) \\
e_{n} & \text { if } \mathrm{G}=\mathrm{SO}(2 \mathrm{n}+1),\end{cases}
\end{aligned}
$$

then the set of simple roots is

$$
\Delta=\left\{\alpha_{1}, \alpha_{2}, \cdots, \alpha_{n}\right\}
$$

indexed as in the Dynkin diagrams $D_{n}$ and $B_{n}$, respectively.

Now let $\theta=\Delta-\left\{\alpha_{n}\right\}$. Let $\tilde{\alpha}_{n}=\left\langle\rho_{\theta}, \alpha_{n}^{\vee}\right\rangle^{-1} \rho_{\theta}$, where $\rho_{\theta}$ is the half sum of all the elements of $\Phi^{+}-\sum_{\theta}$ and $\alpha_{n}^{\vee}=2 \alpha_{n} /\left\langle\alpha_{n}, \alpha_{n}\right\rangle$. By direct computation, 
$\tilde{\alpha}_{n}=1 / 2\left(e_{1}+e_{2}+\cdots+e_{n}\right)$ which is also the nth fundamental weight of $\mathrm{G}$. When Shahidi [9] defined the L-functions at $s$, he identified $s$ with $s \tilde{\alpha}_{n} \in \mathfrak{a}_{\theta, \mathbb{C}}^{*}$. For any $\theta_{*} \subset \theta$, we can get a partition of $\left\{e_{1}, e_{2}, \ldots, e_{n}\right\}$ such that

$$
\begin{aligned}
& e_{1}+\cdots+e_{n_{1}}, \\
& e_{n_{1}+1}+\cdots+e_{n_{2}}, \\
& \cdots \\
& e_{n_{r-1}+1}+\cdots+e_{n_{r}}
\end{aligned}
$$

where $e_{n_{r}}=e_{n}$, is a basis of $\mathfrak{a}_{\theta_{*}, \mathbb{C}}^{*}$. If $\nu \in \mathfrak{a}_{\theta_{*}, \mathbb{C}}^{*}$, we shall simply write

$$
\nu=\left(s_{1}, s_{2}, \ldots, s_{r}\right)
$$

for some $s_{i} \in \mathbb{C}, 1 \leq i \leq r$. In particular,

$$
s \tilde{\alpha}_{n}=(s / 2, s / 2, \ldots, s / 2),
$$

if we embed $\mathfrak{a}_{\theta, \mathbb{C}}^{*}$ into $\mathfrak{a}_{\theta_{*}, \mathbb{C}}^{*}$ in the canonical way.

It is well known that $M_{\theta} \simeq G L(n)$. Let $\pi$ be an irreducible unitary generic representation of $M_{\theta}$, then the classification result on the unitary dual of $G L(n)$, due to Vogan [12] and Tadic [10], says that

$$
\pi \simeq \operatorname{Ind}_{M N}^{G L(n)}\left(\delta_{1} \otimes \cdots \otimes \delta_{m}\right) \otimes q^{\left\langle\nu, H_{M}(\cdot)\right\rangle} \otimes 1_{N},
$$

where $\delta_{i}$ is an irreducible discrete representation of $G L\left(n_{i}\right), \nu=\left(v_{1}, v_{2}, \cdots\right.$, $\left.v_{m}\right), v_{i}$ is a real number and $\left|v_{i}\right|<1 / 2$, for $1 \leq i \leq m, n=n_{1}+\cdots+n_{m}$, $M \simeq G L\left(n_{1}\right) \times \cdots \times G L\left(n_{r}\right)$ and $N$ is the unipotent radical of the standard parabolic subgroup of $\mathrm{G}$ corresponding to $\mathrm{M}$.

For such a $\pi$ in the above, let

$$
e(\pi)=2 \inf \left\{1 / 2-\left|v_{i}\right| ; 1 \leq i \leq m\right\} .
$$

Theorem 4. Let $F$ be a local field of characteristic zero. When $F$ is a $p$ adic field, we let $G=S O(2 n)$ or $S 0(2 n+1)$ and assume that Assumption A holds for $G$. When $F$ is an archimedean field, we let $G=S 0(2 n)$. Let $\theta=\Delta-$ $\left\{\alpha_{n}\right\}$, and $w_{0}=w_{l} w_{l, \theta}$. Let $\pi$ be an irreducible unitary generic representation of $M_{\theta}$. Then $N\left(s \tilde{\alpha}_{n}, \pi, \theta, w_{0}\right)$ is holomorphic and nonvanishing at $s$ for $\operatorname{Re}(s)>-e(\pi)$.

We need the following lemma.

Lemma 3. Let $w \in W$ such that $w \theta \subset \Delta$.

(1) Let $G=S O(2 n)$. Suppose that $\theta$ dose not contain $\alpha_{n}$ or $\alpha_{n-1}$. Then no subset $\theta_{i}$ of $\Delta$ in the decomposition described in Proposition 1 can contain both $\alpha_{n}$ and $\alpha_{n-1}$. 
(2) Let $G=S O(2 n+1)$. Suppose that $\alpha_{n} \notin \theta$. Then no subset $\theta_{i}$ of $\Delta$ in the decomposition described in Proposition 1 can contain $\alpha_{n}$.

Proof. 1) Let $\mathrm{G}=\mathrm{SO}(2 \mathrm{n})$. Suppose that the lemma is false. So we can assume that $\theta_{k}$ dose not contain both $\alpha_{n-1}$ and $\alpha_{n}$ for every $k<i$, but $\theta_{i}$ contains both of them. Remember that $\theta_{i}=w_{i} \theta_{i-1}, w_{i}=w_{l, \Omega_{i-1}} w_{l, \theta_{i-1}}$ and $w_{l, \theta_{i-1}} \theta_{i-1}=-\theta_{i-1}, w_{l, \Omega_{i-1}} \Omega_{i-1}=-\Omega_{i-1}$. If both $\alpha_{n}$ and $\alpha_{n-1}$ are not in $\theta_{i-1}$, then at least one of them can not be in $\theta_{i}$. Now suppose that exactly one of $\alpha_{n-1}$ and $\alpha_{n}$ is in $\theta_{i-1}$, say $\alpha_{n-1} \in \theta_{i-1}$ but $\alpha_{n} \notin \theta_{i-1}$. Suppose that $\Omega_{i-1}=\theta_{i-1} \cup\{\alpha\}$. If $\alpha \neq \alpha_{n}$, then $\theta_{i}$ can not contain both $\alpha_{n-1}$ and $\alpha_{n}$, since $\theta_{i} \subset \Omega_{i-1}$. So $\alpha=\alpha_{n}$. Since

$$
\begin{aligned}
w_{l, \theta_{i-1}} \theta_{i-1} & =-\theta_{i-1}, \quad \text { and } \\
w_{l, \Omega_{i-1}}\left\{\alpha_{n-1}, \alpha_{n}\right\} & =\left\{-\alpha_{n-1},-\alpha_{n}\right\},
\end{aligned}
$$

$\theta_{i}$ can not contain both $\alpha_{n-1}$ and $\alpha_{n}$ which contradicts our assumption on $\theta_{i}$ at the beginning of the proof.

2) For $\mathrm{G}=\mathrm{SO}(2 \mathrm{n}+1)$, we assume that $\alpha_{n} \notin \theta_{i-1}$ and $\alpha_{n} \in \theta_{i}$. Then $\alpha_{n} \in \Omega_{i-1}$. Since the longest element $w_{l, \Delta}$ sends every root $\alpha$ to $-\alpha$, we have $\theta_{i}=w_{l, \Omega_{i-1}} w_{l, \theta_{i-1}} \theta_{i-1}=\theta_{i-1}$. This is a contradiction.

Remark. By this Lemma and [8], we see that every $l\left(r_{\theta_{i}}\right)=1$ for $1 \leq i \leq$ $n-1$, where $n$ is the length of $w$. Therefore Conjecture A is true for the L-function $L\left(\nu_{i}, \pi_{i}, \theta_{i}, w_{i}\right), 1 \leq i \leq n-1$. It is clear that $m=1$ for the $m$ in the Theorem 3 .

Proof of Theorem 4. Let $\theta_{*} \subset \theta$ such that

$$
M_{\theta_{*}} \simeq G L\left(n_{1}\right) \times \cdots \times G L\left(n_{m}\right) .
$$

Put

$$
\begin{gathered}
\delta=\delta_{1} \otimes \cdots \otimes \delta_{m} \\
\lambda(s)=\nu+s \tilde{\alpha}_{n}=\left(v_{1}+s / 2, \cdots, v_{m}+s / 2\right) .
\end{gathered}
$$

Since the normalized intertwining operators are compatible with the isomorphic maps of inducing in stages, we see that $N\left(s \tilde{\alpha}_{n}, \pi, \theta, w_{0}\right)$ is holomorphic and nonvanishing at $s$ if and only if $N\left(\lambda(s), \delta, \theta_{*}, w_{0}\right)$ is holomorphic and nonvanishing at $s$.

By Theorem 3 and the remark following Lemma 3, we only need to check that

$$
\left\langle\operatorname{Re} \lambda(s), \alpha^{\vee}\right\rangle>-1
$$

for every $\alpha \in \Phi_{r}^{+}\left(\theta_{*}, w_{0}\right)$. 
If $\alpha=e_{j_{1}}-e_{j_{2}}$, then

$$
\left\langle\operatorname{Re} \lambda(s), \alpha^{\vee}\right\rangle=v_{j_{1}}+\operatorname{Re}(s / 2)-\left(v_{j_{2}}+\operatorname{Re}(s / 2)\right)=v_{j_{1}}-v_{j_{2}},
$$

so $\left\langle\operatorname{Re} \lambda(s), \alpha^{\vee}\right\rangle>-1$, since $\left|v_{i}\right|<1 / 2$, for $1 \leq i \leq m$.

If $\alpha=e_{j_{1}}+e_{j_{2}}$, then

$$
\begin{aligned}
\left\langle\operatorname{Re} \lambda(s), \alpha^{\vee}\right\rangle & =\operatorname{Re}(s)+v_{j_{1}}+v_{j_{2}} \\
& >-\left(1 / 2-\left|v_{j_{1}}\right|\right)-\left(1 / 2-\left|v_{j_{2}}\right|\right)+v_{j_{1}}+v_{j_{2}} \\
& =-1+\left|v_{j_{1}}\right|+\left|v_{j_{2}}\right|+v_{j_{1}}+v_{j_{2}} \\
& \geq-1,
\end{aligned}
$$

since Re $s>-e(\pi)$.

If $\alpha=e_{i}$ (this happens, only if $\mathrm{G}=\mathrm{SO}(2 \mathrm{n}+1)$ ), then

$$
\left\langle\operatorname{Re} \lambda(s), \alpha^{\vee}\right\rangle=v_{i}+\operatorname{Re}(s / 2)>-1,
$$

also by $\operatorname{Re} s>-e(\pi)$.

\section{References}

[1] J. Arthur, Intertwining operators and residues I, Weighted Characters, J. Funct. Anal., 84 (1989), 19-84.

[2] W. Casselman, Introduction to the theory of admissible representations of p-adic reductive groups, preprint.

[3] W. Casselman and F. Shahidi, Irreducibility of standard modules for generic representations, the case of classical groups, preprint.

[4] R. Langlands, On the functional equation satisfied by Eisenstein series, LN 544, Springer Verlag, 1976.

[5] C. Moeglin and J-L. Waldspurger, Le Spectre Résiduel de $G L(n)$, Ann. Scient. Éc. Norm. Sup., 4-e série, 22 (1989), 605-674.

[6] C. Moeglin and J-L. Waldspurger, Spectral decomposition and Eisenstein series, Cambridge University Press, 1995.

[7] F. Shahidi, A Proof of Langlands' conjecture on plancherel measures; complementary series for p-adic groups, Annals of Mathematics, 132 (1990), 273-330.

[8] - On the Ramanujan conjecture and finiteness of poles for certain L-functions, Annals of Mathematics, 127 (1988), 547-584.

[9] _ On certain L-functions, American J. Math., 103(2), 297-355.

[10] M. Tadic, Classification of unitary representations of irreducible representations of general linear group, Non-archimedean case, Ann. Scient. Ec. Norm. Sup., 19 1989, 335-382.

[11] J. Tate, Number theory background, Proceedings of Symposia in Pure Mathematics, $\mathbf{3 3}(\mathbf{2}), 3-27$. 
[12] D. Vogan, The unitary dual of $G L(n)$ over an archimedean field, Inv. Math., 83 (1986), 449-505.

Received April 19, 1996 and revised October 30, 1996. This research was supported by the postdoctoral fellowship of 1994-1995, of Mathematical Sciences Research Institute at Berkeley.

PuRdue UNIVERSITY

West LAFAyETTE, IN 47907

E-mail address: yz@math.purdue.edu 